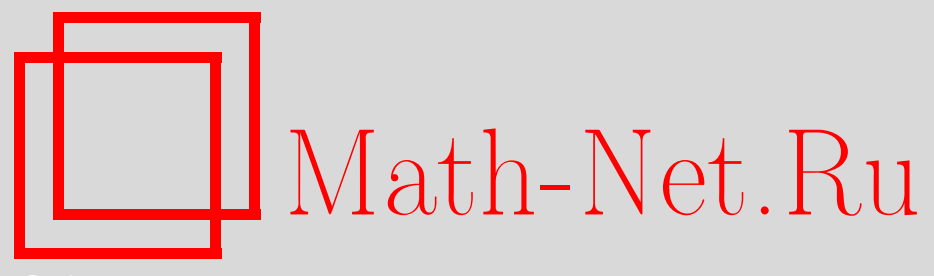

С. Симич, Некоторые свойства целых функций с неотрицательными коэффициентами Тейлора, Матем. заметки, 2007, том 81, выпуск 5, 760-765

DOI: https://doi.org/10.4213/mzm3720

Использование Общероссийского математического портала Math-Net.Ru подразумевает, что вы прочитали и согласны с пользовательским соглашением http://www.mathnet.ru/rus/agreement

Параметры загрузки:

IP: 34.239 .49 .27

26 апреля 2023 г., 12:28:52

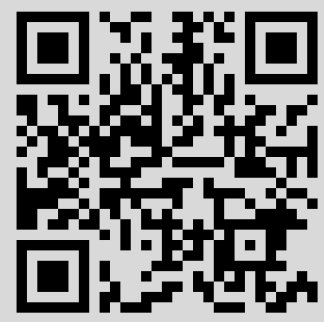


Том 81 выпуск 5 май 2007

УДК 517.547 .2

\section{Некоторые свойства целых функций \\ с неотрицательными коэффициентами Тейлора \\ С. Симич}

Пусть $f$ - целая функция произвольного конечного порядка с неотрицательными коэффициентами Тейлора. Доказано, что ее сужение на положительную часть действительной оси принадлежит классу Хаана Г. Также показано, что $f / f^{\prime}$ - медленно меняющаяся функция Берлинга.

Библиография: 7 названий.

1. Введение. Класс трансцендентных целых функций конечного порядка $\rho, 0 \leqslant$ $\rho<\infty$, с неотрицательными коэффициентами Тейлора будем обозначать через $A_{\rho}$.

В этой статье доказываются некоторые свойства этого класса, которые представляют интерес в отдельных разделах действительного или комплексного анализа, а также в теории экстремальных значений.

Сначала приведем некоторые определения, которые нам понадобятся в дальнейшем.

ОПРЕДЕЛЕНИЕ 1. Положительная измеримая функция $a(\cdot)$ называется регулярно меняющейся с индексом $\mu \in \mathbb{R}$, если асимптотическое соотношение

$$
\frac{a(t x)}{a(x)} \rightarrow t^{\mu}, \quad x \rightarrow \infty,
$$

выполняется при любом $t>0$.

Кроме того, соотношение (1) выполняется равномерно на любом компактном $t$-множестве на полуоси $(0, \infty)$ (см. $[1 ;$ с. 6$])$.

Теория регулярной вариации в настоящее время достаточно хорошо развита и находит многочисленные применения в анализе, теории вероятностей, теории чисел и т.д. (см. [1], [2]).

ОПРЕДЕЛЕНиЕ 2. Измеримая функция $b(\cdot)$ называется медленно меняющейся функиией Берлинга, если $b(x)=o(x), x \rightarrow \infty$, и

$$
\forall t \in \mathbb{R} \quad \frac{b(x+t b(x))}{b(x)} \rightarrow 1, \quad x \rightarrow \infty,
$$

(см. [1; с. 120-122]).

Класс таких функций используется для построения обобщений тауберовой теоремы Винера (см. [3]).

(C) С. Симич, 2007 
ОПРЕДЕЛЕНИЕ 3. Класс Г состоит из неубывающих и непрерывных справа функций $c(\cdot)$, для которых существует такая произвольная измеримая функция $b(\cdot)$, что

$$
\forall t \in \mathbb{R} \quad \frac{c(x+t b(x))}{c(x)} \rightarrow e^{t}, \quad x \rightarrow \infty
$$

(cM. [1; c. 174-180]).

Если $c \in \Gamma$ имеет произвольную функцию $b$, то будем писать $c \in \Gamma(b)$.

Класс Г чрезвычайно важен в теории экстремальных значений (см. [4], [5]).

2. Основные результаты. Основной результат этой статьи состоит в следующем.

ТЕОРема 1. Для любой трансцендентной $f \in A_{\rho}$

$$
\lim _{r \rightarrow \infty} \frac{f(r) f^{\prime \prime}(r)}{\left(f^{\prime}(r)\right)^{2}}=1
$$

независимо от порядка $\rho$.

Имеет место следующее простое следствие этой теоремы.

Теорема 2. Для $f \in A_{\rho}$ определим

$$
\widetilde{f}(r):=\frac{f(r)}{f^{\prime}(r)} .
$$

Тогда $\widetilde{f}$ является медленно меняющейся функцией Берлинга, т.е.

$$
\frac{\tilde{f}(r+t \tilde{f}(r))}{\widetilde{f}(r)} \rightarrow 1, \quad r \rightarrow \infty .
$$

Отсюда следует асимптотическая эквивалентность типа Харди для производной $n$-го порядка (см. [6]).

Теорема 3. Для $f \in A_{\rho}$ выполняется соотношение

$$
f^{(n)}(r) \sim \frac{f(r)}{(\widetilde{f}(r))^{n}}, \quad n \in \mathbb{N}, \quad r \rightarrow \infty .
$$

Наконец, имеет место следующее утверждение.

Теорема 4. Если $f \in A_{\rho}$, mo $f \in \Gamma(\widetilde{f})$. Следовательно,

$$
\forall t \in \mathbb{R} \quad \frac{f(r+t \tilde{f}(r))}{f(r)} \rightarrow e^{t}, \quad r \rightarrow \infty .
$$

3. Доказательства. Для $f \in A_{\rho}$ введем обозначение $T(r):=f(r) f^{\prime \prime}(r) /\left(f^{\prime}(r)\right)^{2}$. Сначала докажем, что

$$
\liminf _{r \rightarrow \infty} T(r) \geqslant 1
$$

Если

$$
f(z):=\sum a_{n} z^{n}, \quad a_{n} \geqslant 0
$$


то введем обозначение

$$
f_{0}(z):=f(z), \quad f_{1}(z):=z f^{\prime}(z)=\sum n a_{n} z^{n}, \quad f_{2}(z):=z f_{1}^{\prime}(z)=\sum n^{2} a_{n} z^{n} .
$$

Тогда

$$
T(r)=\frac{f_{0}(r) f_{2}(r)}{\left(f_{1}(r)\right)^{2}}-\frac{f_{0}(r)}{f_{1}(r)}
$$

и при $r>0$

$$
\frac{f_{0}(r) f_{2}(r)}{\left(f_{1}(r)\right)^{2}}>1
$$

в силу неравенства Коши [7; с. 28].

Итак, при $r>0$ имеем

$$
r\left(\frac{f_{0}(r)}{f_{1}(r)}\right)^{\prime}=1-\frac{f_{0}(r) f_{2}(r)}{\left(f_{1}(r)\right)^{2}}<0,
$$

откуда следует, что функция $f_{0} / f_{1}$ монотонно убывает и приближается к нулю в пределе; в противном случае, существует $a>0$ такое, что $\forall r>0 \quad f_{0}(r) / f_{1}(r)>a$. Следовательно, $f^{\prime}(r) / f(r)<1 / a r$, и после интегрирования мы получаем $f(r)=O\left(r^{1 / a}\right)$. Отсюда следует, что, в этом случае $f$ является полиномом, а не трансцендентной функцией.

Следовательно,

$$
\liminf _{r \rightarrow \infty} T(r) \geqslant 1
$$

Докажем, что

$$
\limsup _{r \rightarrow \infty} T(r) \leqslant 1 .
$$

Доказательство этого утверждения основано на предложениях 1 и 2.

ПреДЛОЖЕНИЕ 1. Пустъ целая функиия $g$ конечного порядка $\rho$ удовлетворяет соотношениям

$$
M(r):=\max _{|z|=r}|g(z)|, \quad M_{1}(r):=\max _{|z|=r}\left|g^{\prime}(z)\right|
$$

Тогда

$$
\limsup _{r \rightarrow \infty} \frac{\log \left(r M_{1}(r) / M(r)\right)}{\log r}=\rho
$$

(см. $[2 ;$ c. 13]).

ПРЕДЛОЖЕНИЕ 2. Пусть $h$ - положительная функиия, обладающая свойством

$$
\limsup _{x \rightarrow \infty} \frac{\log h(x)}{\log x}=\rho, \quad 0 \leqslant \rho<\infty .
$$

Тогда существует регулярно меняющаяся функиия $k \in K_{\rho}$ mакая, ито $h(x) \leqslant k(x)$ $u$

$$
\limsup _{x \rightarrow \infty} \frac{k(x)}{h(x)}=1 .
$$


Это предложение известно как теорема об аппроксимации регулярно меняющейся функцией (см. [1; с. 81]).

Введем обозначения

$$
t_{0}(r):=\frac{f_{1}(r)}{f_{0}(r)}, \quad t_{1}(r):=\frac{f_{2}(r)}{f_{1}(r)} .
$$

Тогда имеем

$$
T(r)=\frac{t_{1}(r)}{t_{0}(r)}-\frac{1}{t_{0}(r)} .
$$

Простым следствием предложения 1 является следующая лемма.

Лемма 1. Если $f \in A_{\rho}, m o$

$$
\limsup _{r \rightarrow \infty} \frac{\log t_{0}(r)}{\log r}=\rho .
$$

ДоказАтельство. Так как $f \in A_{\rho}$ можно представить как

$$
f(z)=\sum a_{n} z^{n}, \quad a_{n} \geqslant 0,
$$

для $|z|=r$ получаем

$$
|f(z)|=\left|\sum a_{n} z^{n}\right| \leqslant \sum a_{n}|z|^{n}=f(r),
$$

т.е. $M(r)=f(r)$ и, аналогично, $M_{1}(r)=f^{\prime}(r)$.

Теперь, объединяя лемму 1 и предложение 2 , получим, что для $f \in A_{\rho}$ существует регулярно меняющаяся функция $k \in R_{\rho}$ такая, что

$$
t_{0}(r) \leqslant k(r), \quad \limsup _{r \rightarrow \infty} \frac{k(r)}{t_{0}(r)}=1 .
$$

Таким образом, для произвольного $C>1$ имеем

$$
\frac{k(r)}{C}<t_{0}(r) \leqslant k(r), \quad r>r_{0}(C),
$$

и, следовательно, при любом $t>1$

$$
\frac{k(t r)}{C}<t_{0}(t r) \leqslant k(t r), \quad r>r_{0}(C) .
$$

Объединяя (5) и (6), получим

$$
\frac{t_{0}(t r)}{t_{0}(r)}<C \frac{k(t r)}{k(r)}, \quad r>r_{0}(C),
$$

т.е., применяя оценки Поттера [1; с. 25], в силу определения 1 окончательно получаем, что неравенство

$$
\frac{t_{0}(t r)}{t_{0}(r)}<C^{2} t^{\rho+1}, \quad r>r_{1}(C),
$$

выполняется при каждом $t>1$. 
Приведем еще одно важное утверждение.

ЛЕмма 2. При любых $t>1, r>0$ имеем

$$
t_{i}(r) \log t<\log \frac{f_{i}(t r)}{f_{i}(r)}<t_{i}(t r) \log t, \quad i=0,1 .
$$

ДокАЗАТЕЛЬСтво. Поскольку $t_{i}(r)$ монотонно возрастает при $r>0$, имеем

$$
t_{i}(r) \log t=t_{i}(r) \int_{r}^{t r} \frac{d u}{u}<\int_{r}^{t r} t_{i}(u) \frac{d u}{u}=\log \frac{f_{i}(t r)}{f_{i}(r)}<t_{i}(t r) \log t .
$$

Теперь, объединяя это утверждение с леммой 2 и соотношением (7) и учитывая тот факт, что $t_{0}=f_{1} / f_{0}$, получим

$$
\begin{aligned}
t_{1}(r) \log t & <\log \frac{f_{1}(t r)}{f_{1}(r)}<\log \left(C^{2} t^{\rho+1}\right)+\log \frac{f_{0}(t r)}{f_{0}(r)}<\log \left(C^{2} t^{\rho+1}\right)+t_{0}(t r) \log t \\
& <\log \left(C^{2} t^{\rho+1}\right)+\left(C^{2} t^{\rho+1} \log t\right) t_{0}(r), \quad r>r_{1}(C) .
\end{aligned}
$$

Поскольку $t_{0}(r) \uparrow \infty$, полагая $t=C^{1 /(\rho+1)}$ в $(8)$, получим

$$
\frac{t_{1}(r)}{t_{0}(r)}<C^{3}+o(1), \quad r \rightarrow \infty .
$$

Так как $C>1$ произвольно, из соотношения (4) окончательно следует, что

$$
\limsup _{r \rightarrow \infty} T(r) \leqslant 1 \text {. }
$$

Лемма доказана.

ДоКАЗАТЕЛЬСТво ТЕОремЫ 2. Имеем $\tilde{f}(r)=r / t_{0}(r)$. Поскольку $t_{0}(r) \uparrow \infty$, получим

$$
\widetilde{f}(r)=o(r), \quad r \rightarrow \infty .
$$

Итак, из теоремы 1 выводим, что

$$
(\tilde{f}(r))^{\prime}=1-T(r) \rightarrow 0, \quad r \rightarrow \infty .
$$

Следовательно,

$$
\widetilde{f}(r+t \widetilde{f}(r))-\widetilde{f}(r)=t \tilde{f}(r)(\widetilde{f})^{\prime}(r+\theta t \widetilde{f}(r)), \quad 0<\theta<1,
$$

т.е.

$$
\frac{\tilde{f}(r+t \tilde{f}(r))}{\widetilde{f}(r)}-1=t(\tilde{f})^{\prime}\left(r\left(1+\frac{\theta t}{t_{0}(r)}\right)\right) .
$$

Теперь очевидно, что правая часть равенства (9) стремится к нулю при $r \rightarrow \infty$ равномерно на любом конечном интервале оси $\mathbb{R}$.

ДОКАЗАТЕЛЬСТво тЕОРЕМЫ 3. Из теоремы 1 следует, что

$$
\frac{f^{\prime \prime}(r)}{f^{\prime}(r)} \sim \frac{f^{\prime}(r)}{f(r)}, \quad r \rightarrow \infty
$$


Однако, если $f \in A_{\rho}$, то также $f^{(m)} \in A_{\rho}, m=1,2, \ldots, n$. Следовательно,

$$
\frac{f^{(n)}(r)}{f^{(n-1)}(r)} \sim \frac{f^{(n-1)}(r)}{f^{(n-2)}(r)} \sim \cdots \sim \frac{f^{\prime \prime}(r)}{f^{\prime}(r)} \sim \frac{f^{\prime}(r)}{f(r)}, \quad r \rightarrow \infty,
$$

T.e.

$$
\frac{f^{(n)}(r)}{f(r)} \sim\left(\frac{f^{\prime}(r)}{f(r)}\right)^{n}, \quad r \rightarrow \infty
$$

откуда следует утверждение теоремы.

ДоКАЗАТЕЛЬСТво тЕОРЕМЫ 4. Из теоремы 2 следует, что

$$
\log \frac{f(r+t \tilde{f}(r))}{f(r)}=\int_{r}^{r+t \tilde{f}(r)} \frac{f^{\prime}(u)}{f(u)} d u=\int_{0}^{t} \frac{\widetilde{f}(r)}{\widetilde{f}(r+u \widetilde{f}(r))} d u \rightarrow t, \quad r \rightarrow \infty .
$$

Теорема доказана.

\section{СПИСОК ЦИТИРОВАННОЙ ЛИТЕРАТУРЫ}

[1] N. H. Bingham, C. M. Goldie, J.L. Teugels, Regular Variation, Encyclopedia of Mathematics and its Applications, 27, Cambridge Univ. Press, Cambridge, 1989.

[2] R. P. Boas, Entire Functions, Academic Press Inc. Publ., New York, 1954.

[3] S. Bloom, "A characterisation of B-slowly varying functions", Proc. Amer. Math. Soc., 54 (1976), 243-250.

[4] S. I. Resnick, Extreme Values, Regular Variation and Point Processes, Applied Probability, 4, Springer-Verlag, New York-Berlin, 1987.

[5] M. R. Leadbetter, G. Lindgren, H. Rootzen, Extremes and Related Properties of Random Sequences and Processes, Springer Series in Statistics, Springer-Verlag, Berlin, 1983.

[6] G. H. Hardy, Orders of Infinity, Cambridge Univ. Press, Cambridge, 1924.

[7] Г. Харди, Дж. Литтлвуд, Г. Полиа, Неравенства, ИЛ, М., 1948.

\section{С. Симич}

Поступило

Математический институт Сербской Академии наук,

20.12 .2005

г. Белград, Сербия

E-mail: ssimic@turing.mi.sanu.ac.yu

Исправленный вариант 08.09.2006 